\title{
Moving toward more transparency
}

\author{
Initiatives to promote transparency in our peer-review process and reproducibility start to take off.
}

$\Lambda$ s of December 2019, Nature Structural \& Molecular Biology and several other Nature journals have implemented a transparent peer review option (https://www.nature.com/ nature-research/editorial-policies/peerreview\#transparent-peer-review). We now offer authors the opportunity to share with readers a peer-review file that includes anonymous referees' reports, authors' responses and our decision letters. This move follows pioneering efforts from EMBO Press and, more recently, Nature Communications. We are excited that this issue of NSMB features the first article whose authors have taken up this offer (https://doi. org/10.1038/s41594-020-0401-0), and we hope more will soon follow suit.

Traditionally, journals have kept peerreview reports and the correspondence between editors and authors confidential, and the peer-review process is thus often seen as a 'black box.' However, calls for more transparency have increased: researchers want to see how publishing decisions are made, and they want assurance that referees and editors act with integrity and without bias. Reviewer reports often provide alternative viewpoints and insights into the merits and limitations of papers, and readers can benefit from such discourse. Opening up the peer-review process also allows the contributions of reviewers to be recognized. After all, reviewers invest a substantial amount of time and effort into assessing a manuscript, and they play a fundamental role in strengthening the work.

We will not edit reviewer reports prior to publication, but we will remove confidential or third-party content, and, in the case of transferred manuscripts, referee reports prepared for journals that do not operate a transparent review process. Importantly, reviewers who now agree to assess manuscripts for us should know that their anonymous reports might be published, if the author chooses this option when the paper is accepted. We of course alert our prospective reviewers when inviting them and hope they will agree that the benefits of enhanced transparency outweigh any perceived drawbacks.

In another initiative to promote transparency, we now ask our authors to share all data central to the main claims of their article as supplementary information or by depositing them in public data repositories. If there are data that can only be shared on request (for example, sensitive human data), authors should explain why in correspondence with the editor and include this explanation in the data availability statement. It has been our long-standing policy that authors are required to make data, materials, code and associated protocols that are necessary to replicate the findings of an article promptly available upon request or to state in the article any restrictions to access. Thus, sharing data upon publication, rather than by request, should not prove overly cumbersome. After all, these data need to be archived and sharing them, either within the article or in public data repositories, allows efficient and publicly accessible storage. This move not only increases transparency but also enables data reanalysis and reuse. Our colleagues at Scientific Data maintain a curated list of recommended data repositories (https://www.nature.com/sdata/policies/ repositories). Deposition of certain types of data remains mandatory (https://www.nature. com/nature-research/editorial-policies/ reporting-standards).

We look forward to seeing our authors and reviewers embracing these editorial initiatives. Feedback from the community is always welcome at nsmb@us.nature.com.

Published online: 8 April 2020

https://doi.org/10.1038/s41594-020-0422-8

\section{Science in the time of COVID-19}

\section{The scientific community is facing challenges due to the pandemic, and we pledge our support.}

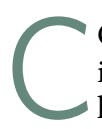
OVID-19 and SARS-CoV-2 need no introduction. The virus has infected hundreds of thousands of people and caused over ten thousand deaths worldwide. In addition, COVID-19 has caused massive disruptions in everybody's lives, with schools and businesses closed, events canceled and travel restricted. Cities, states, even entire countries, have been locked down.

In response to this situation, the scientific community has sprung into action. Researchers have worked around the clock, establishing collaborative efforts and openly sharing their findings and data. The new coronavirus genome was quickly sequenced, structures are being churned out, and clinical and epidemiological data are collected and promptly analyzed. All of this information is essential for guiding public health policies and developing drugs and vaccines.
Funding agencies and publishers have also reacted to these urgent needs, committing to fast and direct access to research on the topic. Springer Nature has made all relevant coronavirus content freely available and encourages early sharing of manuscripts via preprint servers; we also offer support to researchers in depositing their COVID-19 data, at no cost (https://www.springernature.com/gp/ researchers/campaigns/coronavir/research-data).

Clearly, this whirlwind of activity does not apply to all scientists. Most research has been severely hampered by the COVID-19 pandemic, as many universities and research institutes are currently shut down. While public health is paramount, there is concern about the unknown duration and impact of these interruptions.

We editors are fortunate to be able to continue to support our communities while working remotely. We remain committed to scientific excellence and timely editorial processes, but we also understand the challenging circumstances researchers are currently facing. Researchers with teaching duties have had to develop online courses at very short notice, many are also juggling family responsibilities, and we are all concerned for the health and safety of those close to us.

We certainly do not want to cause more anxiety. If you need more time to complete a reviewer report or cannot do it at all, just let us know. If you are unable to perform additional experiments or analyses requested by reviewers, please contact us so we can work together to develop a sensible revision plan and extend timelines as needed. These are stressful times, but we are here to support you.

Published online: 8 April 2020 https://doi.org/10.1038/s41594-020-0423-7 